\title{
Domain structure formation by using Scanning Probe Microscopy: equilibrium polarization distribution and effective piezoelectric response calculations
}

\author{
A.N. Morozovska ${ }^{1 *}$, G.S. Svechnikov ${ }^{1}$, E.I. Shishkin ${ }^{2}$, V.Y. Shur ${ }^{2}$ \\ ${ }^{1}$ V. Lashkaryov Institute of Semiconductor Physics, National Academy of Science of Ukraine, \\ 41, prospect Nauky, 03028 Kyiv, Ukraine \\ ${ }^{2}$ Institute of Physics and Applied Mathematics, Ural State University, \\ Ekaterinburg 620083, Russia \\ *Corresponding author e-mail: morozo@i.com.ua
}

\begin{abstract}
In the paper we adopt the analytical Landau-Ginzburg-Devonshire theory to describe the ferroelectric domain structure formation using Scanning Probe Microscopy. We calculate the effective local piezoresponse of the domain structure within the decoupling approximation using the conventional relation between piezoelectric tensor components and the spontaneous polarization vector. The depth profile of the polarization distribution was derived from the nonlinear Landau-Ginzburg-Devonshire equation. We demonstrate that depending on the material parameters such as the intrinsic domain wall width and probe apex geometry, the shape of the nucleating nanodomains induced by the probe can be either oblate or prolate. The derived analytical expressions for the polarization redistribution caused by the biased probe are valid for both first and second order ferroelectrics.
\end{abstract}

Keywords: effective piezoelectric response, depth profile, nanodomain, scanning probe microscopy.

Manuscript received 18.09.08; accepted for publication 18.03.09; published online 20.03.09.

\section{Overview}

Nanoscale observations of the ferroelectric domain structure and its evolution in external fields have allowed us to obtain direct information on the static wall structure formed after field application, dynamic avalanche time and size distributions, and pinning on individual defects [1-4].

The emergence of the Scanning Probe Microscopy (SPM) based techniques has opened the way for inducing a concentrated electric field within a nanoscale volume of material. Combined with electromechanical response detection, this Piezoresponse Force Microscopy (PFM) approach has been broadly applied to domain imaging and polarization patterning. Piezoresponse force spectroscopy was used to study polarization switching for small volumes. Experimental studies have been complemented by extensive theoretical analysis of domain nucleation mechanisms [5-9] in the approximation of "rigid" spontaneous polarization $\left(+P_{S}\right.$ or $-P_{S}$ inside/outside the domains) assuming atomicallysharp (mathematically infinitely thin) domain walls.
Intrinsic domain wall width is a fundamental parameter that reflects bulk ferroelectric properties and governs the performance of ferroelectric memory devices. Recently [10] we derived closed-form analytical expressions for vertical and lateral Piezoelectric Force Microscopy (PFM) profiles of a single ferroelectric domain wall for the conical and disc models of the tip, beyond point charge and sphere approximations. The analysis takes into account the finite intrinsic width of the domain wall and dielectric anisotropy of the material. These analytical expressions provide insight into the mechanisms of PFM image formation and can be used for quantitative analysis of the PFM domain wall profiles.

In Ref. [11] we consider the interaction of ferroelectric $180^{\circ}$-domain wall polarization with a strongly inhomogeneous electric field of biased force microscope probe within LGD thermodynamic approach for the second order ferroelectrics. The analysis is performed within the framework of the LandauGinzburg-Devonshire (LGD) theory, avoiding the typical limitation of rigid ferroelectric (i.e. infinitely thin domain wall) approximation. Equilibrium shape of the 
initially flat domain wall boundary bends, attracts or repulses from the probe apex, depending on the sign and value of the applied bias. For large tip-wall separations, the probe-induced domain nucleation is possible. The approximate analytical expressions for the equilibrium polarization distribution are derived using the direct variational method. The expressions provide insight into how the surface polarization distribution depends on the wall's finite-width, correlation and depolarization effects, electrostatic potential distribution of the probe and ferroelectric material parameters.

In the paper we adopt the analytical LGD theory to describe the domain structure formation using SPM. We calculate the effective local piezoresponse and study the depth profile of the polarization distribution. We demonstrate that depending on the material parameters such as the intrinsic domain wall width, the shape of the equilibrium domains, induced by the probe, can be either oblate or prolate. The derived analytical expressions are valid for both first and second order ferroelectrics.

\section{The problem statement}

The resolution and probed volume in PFM is determined by the structure of electric and elastic fields inside the material. In general, the calculation of the fields in the material requires the solution to a coupled problem, which is currently available only for a transversally isotropic case and is also limited to the electric field produced in the contact area.

Here we use a simplified approach suggested by Felten et al. [12] and Scrymgeour and Gopalan [13] that is based on the solution of a decoupled electromechanical problem. In this case, the electric field in the material is calculated using a rigid electrostatic model (no piezoelectric coupling); the strain or stress field is calculated using constitutive relations for a piezoelectric solid, and the displacement field is evaluated using an appropriate Green's function for an isotropic or anisotropic solid. Hereinafter, we consider semi-infinite ferroelectrics.

In decoupled approximation, the surface displacement $u_{i}(\mathbf{r}, \mathbf{y})$ at location $\mathbf{y}$ induced by the biased probe at position $\mathbf{r}$ is:

$$
\begin{aligned}
& u_{i}(\mathbf{r}, \mathbf{y})=\int_{-\infty}^{\infty} d \xi_{1} \int_{-\infty}^{\infty} d \xi_{2} \int_{0}^{\infty} d \xi_{3} \frac{\partial G_{i j}\left(x-\xi_{1}, y-\xi_{2}, \xi_{3}\right)}{\partial \xi_{k}} \times \\
& \times E_{l}(\xi) \cdot c_{k j m n} d_{l n m}\left(y_{1}+\xi_{1}, y_{2}+\xi_{2}, \xi_{3}\right)
\end{aligned}
$$

Where the electric field $E_{k}(\mathbf{r})=-\partial \varphi / \partial x_{k}$ is produced by the tip in the point $\mathbf{r}=(x, y, z)$ of the sample, $d_{k l j}(\mathbf{r})$ are the stress piezoelectric tensor components representing material properties (ideal image), $c_{k j m n}$ are stiffness tensor components. Coordinate systems $\mathbf{x}$ and $\xi$ are linked to the probe apex, coordinates $\mathbf{y}=\left(y_{1}, y_{2}, z\right)$ is the probe apex position in the sample coordinate system y. For most inorganic ferroelectrics, the elastic properties are weakly dependent on orientation. Hence, material can be approximated as elastically isotropic. The corresponding Green's tensor $G_{i j}(\mathbf{r}, \xi)$ for an elastically isotropic half-plane is given by Lur'e [14] and Landau and Lifshitz [15].

If the sample is uniform on the scale of the penetration depth of electric field, i.e., $c_{j l m n} d_{m n k}(x, y, z) \approx c_{j l m n} d_{m n k}(x, y)$, vertical surface displacement below the tip, i.e., vertical PFM signal, can be rewritten as

$$
\begin{aligned}
& u_{3}(\mathbf{0}, \mathbf{y})=\int_{-\infty}^{\infty} d_{m n k}(\mathbf{y}-\xi) \times \\
& \times\left(\int_{z=0}^{\infty} c_{j l m n} E_{k}\left(-\xi_{1},-\xi_{2}, z\right) \frac{\partial}{\partial \xi_{l}} G_{3 j}\left(\xi_{1}, \xi_{2}, z\right) d z\right) d \xi_{1} d \xi_{2},
\end{aligned}
$$

i.e., as a convolution of a function describing the spatial distribution of material properties, $d_{m n k}(\mathbf{r})$, and a resolution function related to probe parameters (integral in parenthesis).

The spontaneous polarization $P_{3}(\mathbf{r})$ of ferroelectrics is directed along the polar axis, $z$. The sample is dielectrically isotropic in transverse directions, i.e. permittivities $\varepsilon_{11}=\varepsilon_{22}$, while $\varepsilon_{33}$ value may be different. The dependence of in-plane polarization components on an electric field is linearized as $P_{1,2} \approx-\varepsilon_{0}\left(\varepsilon_{11}-1\right) \partial \varphi(\mathbf{r}) / \partial x_{1,2}$. The conventional relation between piezoelectric coefficients $d_{i j k}=2 \varepsilon_{0} \varepsilon_{i l} Q_{j k l m} P_{m}$ in Voigth notation acquire the explicit form:

$$
\begin{aligned}
& d_{33}=2 \varepsilon_{0} \varepsilon_{33} Q_{11} P_{3}, \quad d_{31}=2 \varepsilon_{0} \varepsilon_{33} Q_{12} P_{3}, \\
& d_{15}=2 \varepsilon_{0} \varepsilon_{11} Q_{44} P_{3} .
\end{aligned}
$$

Where $Q_{i j}$ is electrostriction tensor components in Voigt notation.

The problem for quasi-static electrostatic potential $\varphi(\mathbf{r})$ can be written as:

$$
\left\{\begin{array}{l}
\varepsilon_{33}^{b} \frac{\partial^{2} \varphi}{\partial z^{2}}+\varepsilon_{11}\left(\frac{\partial^{2} \varphi}{\partial x^{2}}+\frac{\partial^{2} \varphi}{\partial y^{2}}\right)=\frac{1}{\varepsilon_{0}} \frac{\partial P_{3}}{\partial z}, \\
\varphi(x, y, z=0)=V_{e}(x, y), \quad \varphi(x, y, z \rightarrow \infty)=0 .
\end{array}\right.
$$

Here we introduced dielectric permittivity of background [16] or reference state [17] as $\varepsilon_{33}^{b}$. Typically $\varepsilon_{33}^{b} \leq 10 ; \varepsilon_{0}$ is the universal dielectric constant.

The electrostatic potential $\varphi(\mathbf{r})$ includes the effects of the depolarization field created by polarization bound charges. The perfect screening of a depolarization field outside the sample is realized by the ambient screening charges, as shown in Fig. 1. 


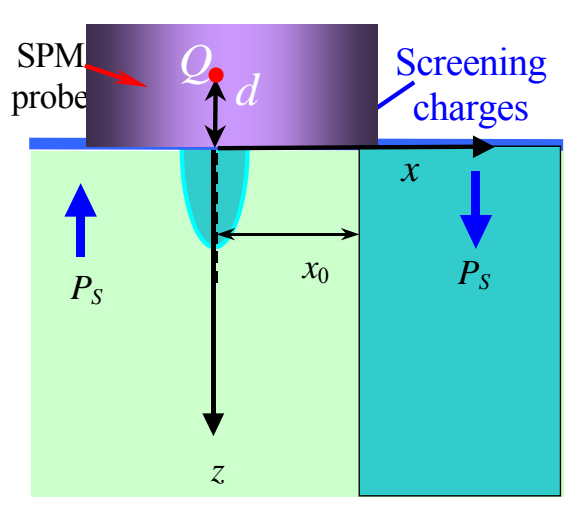

Fig. 1. Schematics of the domain nucleation near ferroelectric $180^{\circ}$-domain wall boundary. The nucleation is caused by the strong inhomogeneous electric field of the biased SPM probe in contact with the sample surface.

The potential distribution produced by the SPM probe on the surface can be approximated as $V_{e}(x, y) \approx V d / \sqrt{x^{2}+y^{2}+d^{2}}$, where $V$ is the applied bias, $d$ is the effective distance determined by the probe geometry [8]. The potential is normalized assuming the condition of perfect electrical contact with the surface, $V_{e}(0,0) \approx V$. In the case of a local point charge model, the probe is represented by a single charge $Q=2 \pi \varepsilon_{0} \varepsilon_{e} R_{0} V\left(\kappa+\varepsilon_{e}\right) / \kappa \quad$ located at distance $d=\varepsilon_{e} R_{0} / \kappa$ for a spherical tip apex with curvature $R_{0}$ $\left(\kappa \approx \sqrt{\varepsilon_{33} \varepsilon_{11}}\right.$ is the effective dielectric constant determined by the "full" dielectric permittivity $\varepsilon_{33}$ in zdirection, $\varepsilon_{e}$ is ambient dielectric constant), or $d=2 R_{0} / \pi$ for a flattened tip represented by a disk of radius $R_{0}$ in contact with the sample surface. In any case, one should take into account that $\frac{Q}{2 \pi \varepsilon_{0}\left(\kappa+\varepsilon_{e}\right) d}=V$.

In the framework of LGD phenomenology, in the absence of pinning centers or for weak pinning of viscous friction type the equilibrium polarization distribution can be found as the solution of the nonlinear equation:

$$
\begin{aligned}
& \alpha P_{3}+\beta P_{3}^{3}+\delta P_{3}^{5}-\xi \frac{\partial^{2} P_{3}}{\partial z^{2}}- \\
& -\eta\left(\frac{\partial^{2} P_{3}}{\partial x^{2}}+\frac{\partial^{2} P_{3}}{\partial y^{2}}\right)=E_{3} .
\end{aligned}
$$

The gradient terms $\xi>0$ and $\eta>0$, expansion coefficients $\delta>0$, while $\beta<0$ for the first order phase transitions or $\beta>0$ for the second order ones. Coefficient $\alpha<0$ in ferroelectric phase. Rigorously, coefficient $\alpha$ should be taken as renormalized by the elastic stress $[18,19] . E_{3}$ is electric field.
The corresponding Fourier-image on transverse coordinates $\{x, y\}$ of electric field normal component $\widetilde{E}_{3}(\mathbf{k}, z)=-\partial \widetilde{\varphi} / \partial z$ is the sum of external (e) and depolarization $(d)$ fields [11]:

$$
\begin{aligned}
& \widetilde{E}_{3}(\mathbf{k}, z)=\widetilde{E}_{3}^{e}(\mathbf{k}, z)+\widetilde{E}_{3}^{d}(\mathbf{k}, z), \\
& \widetilde{E}_{3}^{e}(\mathbf{k}, z)=\widetilde{V}_{e}(\mathbf{k}) \frac{\cosh \left(k(h-z) / \gamma_{b}\right)}{\sinh \left(k h / \gamma_{b}\right)} \frac{k}{\gamma_{b}}, \\
& \widetilde{E}_{3}^{d}(\mathbf{k}, z)=\left(\int_{0}^{z} d z^{\prime} \frac{\widetilde{P}_{3}\left(\mathbf{k}, z^{\prime}\right)}{\varepsilon_{0} \varepsilon_{33}^{b}} \cosh \left(k z^{\prime} / \gamma_{b}\right) \times\right. \\
& \times \frac{\cosh \left(k(h-z) / \gamma_{b}\right)}{\sinh \left(k h / \gamma_{b}\right)} \frac{k}{\gamma_{b}}+ \\
& +\int_{z}^{h} d z^{\prime} \frac{\widetilde{P}_{3}\left(\mathbf{k}, z^{\prime}\right)}{\varepsilon_{0} \varepsilon_{33}^{b}} \cosh \left(k\left(h-z^{\prime}\right) / \gamma_{b}\right) \times \\
& \left.\times \frac{\cosh \left(k z / \gamma_{b}\right)}{\sinh \left(k h / \gamma_{b}\right)} \frac{k}{\gamma_{b}}-\frac{\widetilde{P}_{3}(\mathbf{k}, z)}{\varepsilon_{0} \varepsilon_{33}^{b}}\right) .
\end{aligned}
$$

Here $\gamma_{b}=\sqrt{\varepsilon_{33}^{b} / \varepsilon_{11}}$ is the "bare" dielectric anisotropy factor, $\mathbf{k}=\left\{k_{1}, k_{2}\right\}$ is a spatial wave-vector, its absolute value $k=\sqrt{k_{1}^{2}+k_{2}^{2}}, h$ is the sample thickness. The Fourier-image of polarization is $\widetilde{P}_{3}(\mathbf{k}, z)=\frac{1}{2 \pi} \int_{0}^{\infty} d t \int_{-\infty}^{\infty} d x \int_{-\infty}^{\infty} d y \exp \left(i k_{1} x+i k_{2} y\right) P_{3}(x, y, z)$

The Fourier-image of the electric field potential at the sample surface is $\quad \tilde{V}_{e}(\mathbf{k})=V \widetilde{w}(\mathbf{k}), \quad$ where $\widetilde{w}(\mathbf{k})=\frac{d}{k} \exp (-k d)$. For a transversally homogeneous media, $\varepsilon_{33}^{b}=1$ and static case Eq. (6b) reduces to the expression for a depolarization field obtained by Kretschmer and Binder [20].

For the semi-infinite sample considered hereinafter, initial and boundary conditions for polarization are the following

$$
P_{3}(\mathbf{r}, t \leq 0)=P_{0}(x), \quad \frac{\partial P_{3}}{\partial z}(z=0)=0 .
$$

The condition $\partial P_{3} / \partial z=0$ corresponds to the perfect atomic surface structure without defects or a damaged layer and so one could neglect the surface energy contribution. Polarization distribution $P_{0}(x)$ satisfies Eq. (5) at zero external bias, $V_{e}=0$. For the first order ferroelectrics the $180^{\circ}$-domain wall profile unperturbed by the probe is $P_{0}(x)=$ $=\frac{P_{S} \cdot \sinh \left(\left(x-x_{0}\right) / 2 L_{\perp}\right)}{\sqrt{3\left(2 \alpha_{S}+\beta_{S} P_{S}^{2}\right) /\left(4 \alpha_{S}+\beta_{S} P_{S}^{2}\right)+\sinh ^{2}\left(\left(x-x_{0}\right) / 2 L_{\perp}\right)}}$,
where the correlation length is 
$L_{\perp}=\sqrt{\eta /\left(\alpha+3 \beta P_{S}^{2}+5 \delta P_{S}^{4}\right)}$ and the spontaneous polarization $P_{S}^{2}=\left(\sqrt{\beta^{2}-4 \alpha \delta}-\beta\right) / 2 \delta \quad$ [21]. For the second order ferroelectrics the solution of equation $\alpha P_{0}(x)+\beta P_{0}(x)^{3}-\eta \frac{\partial^{2} P_{0}(x)}{\partial x^{2}}=0$ for the initial flat domain wall profile positioned at $x=x_{0}$ is $P_{0}(x)=P_{S} \tanh \left(\left(x-x_{0}\right) / 2 L_{\perp}\right)$, where the correlation length is $L_{\perp}=\sqrt{-\eta / 2 \alpha}$, and the spontaneous polarization is $P_{S}^{2}=-\alpha / \beta$.

\section{Polarization re-distribution: domain nucleation and wall bending}

To obtain the spatial re-distribution of polarization an arbitrary bias we used the direct variational method [11]. In this method, k-dependent (i.e. coordinate-dependent) part of linearized solution (5) was used as the trial function in the corresponding free energy functional, the bias-dependent amplitude (in volts) was treated as a variational parameter $P_{V}$ (like scalar order parameter in GLD). Allowing for the radial symmetry of the normalized probe potential $\widetilde{w}(k)=d \exp (-k d) / k$, we obtained the dependence of polarization in the form [11]: $P_{3}(\mathbf{r})=P_{0}(x)+p(\mathbf{r})$,

$p(\mathbf{r})=-P_{V} \int_{0}^{\infty} \frac{k^{2}}{\gamma_{b}} d k \cdot J_{0}\left(k \sqrt{x^{2}+y^{2}}\right) \times$

$\times \tilde{w}(k) \frac{s_{2} \exp \left(-s_{1} z\right)-s_{1} \exp \left(-s_{2} z\right)}{\sqrt{\xi\left(\eta k^{2}-2 \alpha_{S}\right)\left(s_{1}^{2}-s_{2}^{2}\right)}}$.

The characteristic equation for eigenvalues $s(k)$ is biquadratic, namely $\left(\varepsilon_{33}^{b} s^{2}-\varepsilon_{11} k^{2}\right)\left(-2 \alpha_{S}-\left(\xi s^{2}-\eta k^{2}\right)\right)=$ $=-\frac{s^{2}}{\varepsilon_{0}}{ }^{1}$, where $\alpha_{S}=\alpha\left(1-\frac{6 L_{\perp}\left(L_{\perp}+d\right)}{\pi\left(\left(L_{\perp}+d\right)^{2}+x_{0}^{2}\right)}\right)$. For

${ }^{1}$ It is seen that for any real values of $k$ values of $s_{1,2}(k)$ are real and the identity is valid:

$s_{1}^{2}-s_{2}^{2}=\frac{\sqrt{\left(1+\varepsilon_{0}\left(k^{2}\left(\varepsilon_{11} \xi+\eta \varepsilon_{33}^{b}\right)-2 \varepsilon_{33}^{b} \alpha_{S}\right)\right)^{2}-4\left(\varepsilon_{0} k\right)^{2} \xi \varepsilon_{11} \varepsilon_{33}^{b}\left(\eta k^{2}-2 \alpha_{S}\right)}}{\varepsilon_{0} \varepsilon_{33}^{b} \xi}$

At the sample surface, $z=0$, Eq. (8), can be simplified as:

$P_{3}(x, y, 0)=P_{0}(x)+\int_{0}^{\infty} \frac{d k \cdot k^{2} \sqrt{\varepsilon_{11} \varepsilon_{0}} J_{0}(k r)\left(\eta k^{2}-2 \alpha_{S}\right)^{-1 / 2} \widetilde{w}(k) \cdot P_{V}(V)}{\sqrt{1+\varepsilon_{0}\left(k^{2}\left(\varepsilon_{11} \xi+\eta \varepsilon_{33}^{b}\right)-2 \varepsilon_{33}^{b} \alpha_{S}\right)+2 \varepsilon_{0} k \sqrt{\xi \varepsilon_{11} \varepsilon_{33}^{b}\left(\eta k^{2}-2 \alpha_{S}\right)}}}$

. For particular case $\xi \rightarrow \infty$ we obtained that $s_{1} \rightarrow 0$ and so

$P_{3}(\mathbf{r}) \approx P_{0}(x)+P_{V} \int_{0}^{\infty} \frac{k^{2}}{\gamma_{b}} d k \frac{J_{0}(k \rho) \widetilde{w}(k) s_{2} \exp \left(-s_{1} z\right)}{\sqrt{\xi\left(\eta k^{2}-2 \alpha_{S}\right)}\left(s_{2}^{2}-s_{1}^{2}\right)} \sim P_{0}(x)+$

$+P_{V} \int_{0}^{\infty} \frac{k^{2}}{\gamma_{b}} d k \frac{J_{0}(k \rho) \widetilde{w}(k) \exp \left(-s_{1} z\right)}{\sqrt{\xi\left(\eta k^{2}-2 \alpha_{S}\right)\left(s_{2}+s_{1}\right)}}$. small $k$ values $s_{1} \approx \sqrt{\frac{1+\left(-2 \alpha_{S}\right) \varepsilon_{0} \varepsilon_{33}^{b}}{\varepsilon_{0} \varepsilon_{33}^{b} \xi}} \quad$ and $s_{2} \approx k \sqrt{\frac{\varepsilon_{11} \varepsilon_{0}\left(-2 \alpha_{S}\right)}{1+\left(-2 \alpha_{S}\right) \varepsilon_{0} \varepsilon_{33}^{b}}}$.

For typical ferroelectric material parameters the inequality $2 \varepsilon_{0} \varepsilon_{33}^{b}|\alpha|<<1$ is valid, since $\varepsilon_{33}^{b} \leq 10$, and so the integration in Eq. (8) can be performed for several particular cases:

$$
P_{3} \approx\left\{\begin{array}{l}
P_{0}(x)+\sqrt{\varepsilon_{11} \varepsilon_{0} /\left(-2 \alpha_{S}\right)}\left(d+z / \gamma_{S}\right) d \cdot P_{V} \\
\frac{\left(\sqrt{\eta /\left(-2 \alpha_{S}\right)}\left(d+z / \gamma_{S}\right)+\left(d+z / \gamma_{S}\right)^{2}+\rho^{2}\right) \sqrt{\left(d+z / \gamma_{S}\right)^{2}+\rho^{2}}}{\xi<-2 \alpha d^{2},} \\
\quad\left(s_{1} \rightarrow \infty\right) \\
P_{0}(x)+\frac{\sqrt{\varepsilon_{11} \varepsilon_{0} /\left(-2 \alpha_{S}\right)} d^{2} \cdot P_{V}}{\left(\sqrt{\eta /\left(-2 \alpha_{S}\right)} d+d^{2}+\rho^{2}\right) \sqrt{d^{2}+\rho^{2}}} \exp \left(-\frac{z}{\sqrt{\varepsilon_{0} \varepsilon_{33}^{b} \xi}}\right), \\
\xi>-2 \alpha d^{2}, \\
\quad\left(s_{1} \rightarrow 0\right) .
\end{array}\right.
$$

Here parameter $\rho=\sqrt{x^{2}+y^{2}}$ has the meaning of radial coordinate,

factor $\gamma_{S}=\sqrt{\frac{1-2 \alpha_{S} \varepsilon_{33}^{b} \varepsilon_{0}}{-2 \alpha_{S} \varepsilon_{11} \varepsilon_{0}}} \approx \sqrt{\frac{1}{-2 \alpha_{S} \varepsilon_{11} \varepsilon_{0}}} \approx \sqrt{\frac{\varepsilon_{33}}{\varepsilon_{11}}}$.

Under the absence of domain wall pinning, the equilibrium domain wall boundary $x_{D W}(y, z)$ can be determined from the condition $P_{3}\left(x_{D W} y, z\right)=0$. Let us postulate that the threshold (or critical) positive and negative biases $V_{t h}^{ \pm}$are required to move the domain wall boundary by overcoming the effect of the lattice constant discreteness $a$. The presence of lattice pinning leads to the appearance of the threshold bias $V_{t h}^{ \pm}$. With the presence of lattice pinning and defects, the amplitude $P_{V}$ can be obtained from equations:

$$
\left\{\begin{array}{l}
P_{V}=0, \quad V_{t h}^{-}<V<V_{t h}^{+}, \\
P_{V}+w_{3} P_{V}^{2}+w_{4} P_{V}^{3}+w_{6} P_{V}^{5}=V, \quad V<V_{t h}^{-} \text {or } V>V_{t h}^{+} .
\end{array}\right.
$$

Parameters

$$
\begin{gathered}
w_{3}\left(x_{0}\right)=-\frac{\left(5 \delta P_{S}^{2}+3 \beta\right) P_{S} x_{0}}{\sqrt{\left(L_{\perp}+d\right)^{2}+x_{0}^{2}}} \frac{\sqrt{-2 \alpha_{S} \varepsilon_{11} \varepsilon_{0}}}{4 \alpha_{S}^{2}\left(L_{\perp}+d\right)}, \\
w_{4}=\frac{\beta \varepsilon_{11} \varepsilon_{0}}{4 \alpha_{S}^{2}\left(L_{\perp}+d\right)^{2}} \text { and } w_{6}=\frac{-\delta\left(\varepsilon_{11} \varepsilon_{0}\right)^{3 / 2}}{8 \alpha_{S}^{3}\left(L_{\perp}+d\right)^{4}} .
\end{gathered}
$$

Positive and negative threshold bias (solid and dashed curves respectively), calculated numerically, via effective distance $d$ are shown in Fig. 2 for $\mathrm{LiNbO}_{3}$ material parameters and $\xi<<-2 \alpha d^{2}$. 


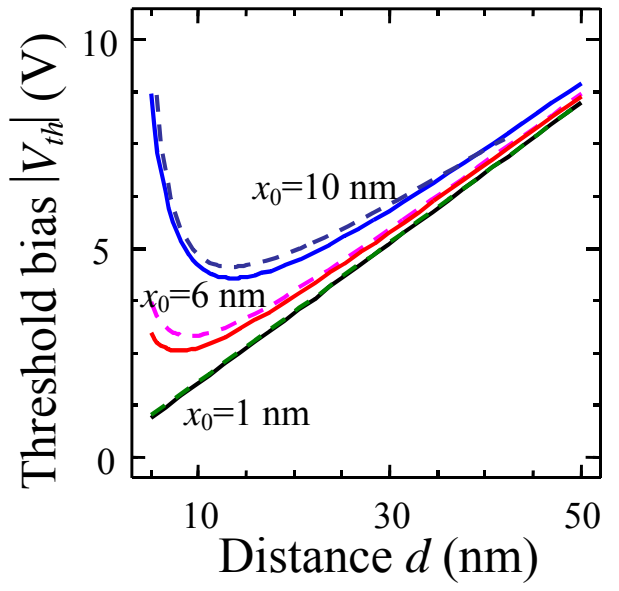

Fig. 2. Absolute values of positive and negative threshold bias (solid and dashed curves, respectively) via effective distance $d$ for $\mathrm{LiNbO}_{3}$ material parameters $\left(\varepsilon_{11}=84, \varepsilon_{33}=30, \varepsilon_{33}^{b} \leq 3\right.$, $\alpha=-2 \cdot 10^{9}, \eta=\xi=10^{-9}$ in SI units (i.e. $L_{\perp}=L_{z}=0.5 \mathrm{~nm}$ ), $P_{s}=$ $0.75 \mathrm{C} / \mathrm{m}^{2}$ ) and different $x_{0}$ values (labels near the curves).
Depth profiles of polarization distribution perturbed by the biased probe can be calculated from Eqs. (8)-(9), where the bias dependence $P_{V}(V)$ is given by nonlinear Eq. (10). The corresponding depth profiles are shown in Fig. 3.

For chosen material constants, effective distance $d=5 \mathrm{~nm}$ and $\xi<<-2 \alpha d^{2}$ characteristic depth $z_{d}$ of domain wall bending is also about $5 \mathrm{~nm}$, since $z \sim \gamma_{S} d$ as anticipated from the first of Eqs. (9). For a chosen polarization distribution the wall attraction to the probe corresponds to positive biases, while the domain wall repulsion from the probe takes place at negative biases.

The domain wall boundary bending by biased probe is observed at distances $\left|x_{0}\right|<L_{\perp}+d$, while far from the wall at distances $\left|x_{0}\right|>L_{\perp}+d$ the probeinduced domain formation appears at bias more than coercive one [11].
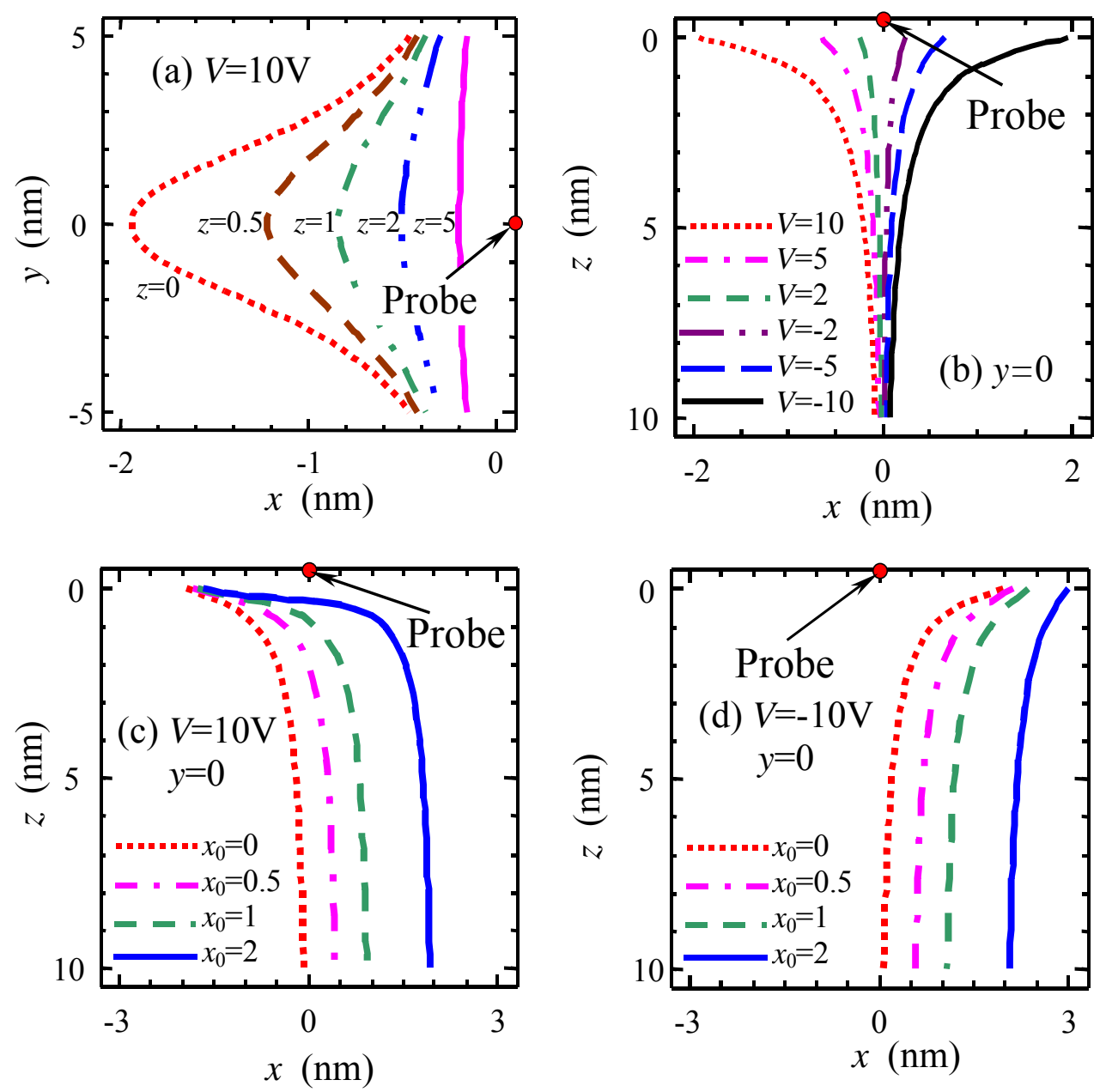

Fig. 3. Depth profiles of the domain wall affected by biased probe. (a) Lateral cross-sections at different depth, marked near curves in $\mathrm{nm}$. (b) Vertical section for different bias (marked in figure in $\mathrm{V}$ ) at a fixed distance between wall and probe $x_{0}=0$. (c, d) The vertical section for different distances between wall and probe (marked in figure in nm) at a fixed voltage $V=$ $10 \mathrm{~V}(\mathrm{c})$ and $V=-10 \mathrm{~V}(\mathrm{~d})$. Material parameters for $\mathrm{LiNbO}_{3}$ are the same as in Fig. 2. Effective distance $d=5 \mathrm{~nm}$. 
The profiles of the probe-induced domain nucleation far from the wall and near the wall, calculated under the condition of small longitudinal gradient coefficient $\xi<<-2 \alpha d^{2}$, are shown in Figs. 4. For chosen material constants, the length of the domain $l \sim \gamma_{S} d$ is determined by the product of anisotropy factor $\gamma_{S}$ on effective charge-surface separation $d$, as anticipated from the first of Eq. (9). It is clear that nucleated domain is oblate (its radius $r>l$ ) for $\gamma_{S}<1$ and prolate $(r<l)$ for $\gamma_{S}>1$.

Under the condition of high longitudinal gradient coefficient $\xi>>-2 \alpha d^{2}$, the length of the domain is determined by depolarization length $\sqrt{\varepsilon_{0} \varepsilon_{33}^{b} \xi}$, as anticipated from the second of Eq. (9). It is clear that nucleated domain is prolate (its radius $r<<l$ ).
Note that the small longitudinal correlation length $l_{C}=\sqrt{\frac{\xi}{\varepsilon_{0}^{-1}-2 \alpha}}<<\sqrt{\frac{\xi}{-2 \alpha}}$ can appear due to the depolarization field that strongly suppresses any fluctuations of the order parameter in polar direction $z$. However, the experimentally measurable intrinsic domain wall width $L_{z}=\sqrt{-\xi / 2 \alpha}$ in $z$-direction typically appeared rather high in lithium niobate, which is possible only at a high longitudinal gradient coefficient $\xi$. Note that the depolarization field drastically decreases with an increase in the domain wall width. Namely, Gopalan et al. [22] recently have shown that an increase in the anti-parallel counter domain wall width from $0.5 \mathrm{~nm}$ to $2-3 \mathrm{~nm}$ lead to a decrease in the coercive field on $2-3$ orders of magnitude. The inequality $\xi>>$ is necessary for prolate domain appearance.
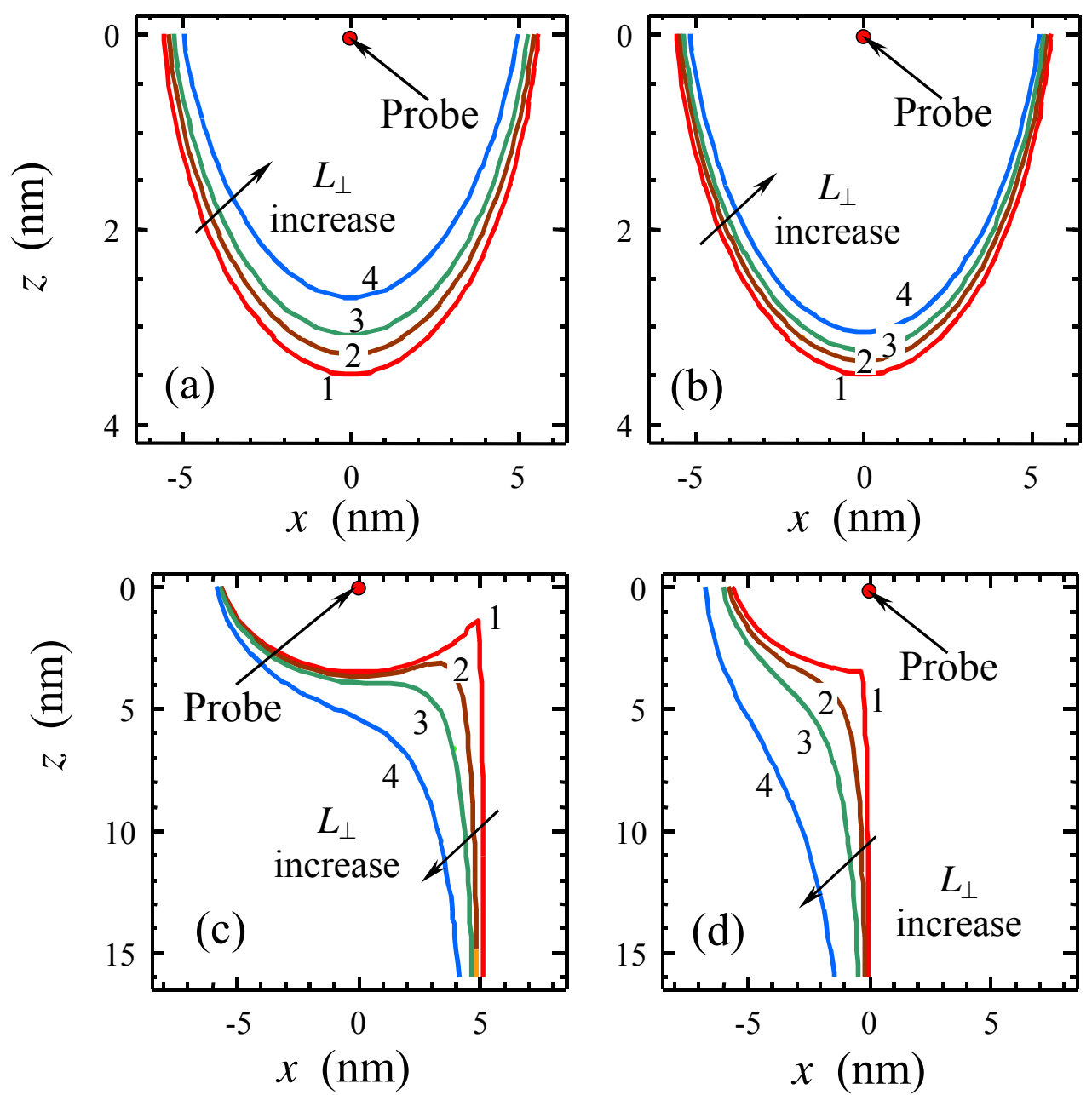

Fig. 4. Vertical cross-sections of probe-induced domain formation near the domain wall for different distances from initial flat wall $x_{0}=\infty, 15,5,0 \mathrm{~nm}$ (panels (a), (b), (c) and (d), respectively). Curves 1, 2, 3, 4 correspond to different values of $L_{\perp}=0$, 0.5, 1, $2 \mathrm{~nm}$. PZT40/60 parameters: $\varepsilon_{11}=497, \varepsilon_{33}=197, \varepsilon_{33}^{b} \leq 10, \alpha=-1.66 \cdot 10^{8} \mathrm{~m} / \mathrm{F}, \beta=1.44 \cdot 10^{8} \mathrm{~m}^{5} /\left(\mathrm{C}^{2} \mathrm{~F}\right), \xi=10^{-9} \mathrm{SI}$ units. Effective distance $d=5 \mathrm{~nm}$, applied bias $V=10 \mathrm{~V}$. 


\section{Effective piezoresponse calculations}

The tensorial object transfer function (OTF) $\widetilde{W}_{i j k l}(\mathbf{q})$ is defined as a Fourier-image of the resolution function, namely:

$\widetilde{W}_{i j k l}(\mathbf{q})=c_{k j m n} \int_{-\infty}^{\infty} d k_{1} \int_{-\infty}^{\infty} d k_{2} \int_{0}^{\infty} d \xi_{3} \widetilde{G}_{i m, n} \times$

$\times\left(q_{1}-k_{1}, q_{2}-k_{2}, \xi_{3}\right) \widetilde{E}_{l}\left(k_{1}, k_{2}, \xi_{3}\right)$.

For the dielectrically transversely isotropic ferroelectric $\widetilde{W}_{i j k}(\mathbf{q})$ depends only on the absolute value of the wave vector $q=|\mathbf{q}|$. The rotationally invariant OTF is derived as

$F_{3}(q)=\widetilde{W}_{333}(q) d_{33}+\widetilde{W}_{313}(q) d_{31}+\widetilde{W}_{351}(q) d_{15}$

Using relations (3), Eq. (13) acquires the form:

$\widetilde{F}_{3}(k)=2 \varepsilon_{0}\left(\widetilde{W}_{333}(k) \varepsilon_{33} Q_{11}+\widetilde{W}_{313}(k) \varepsilon_{33} Q_{12}+\right.$

$\left.+\widetilde{W}_{351}(k) \varepsilon_{11} Q_{44}\right) \widetilde{P}_{3}(\mathbf{k}, 0)$.

$\widetilde{P}_{3}(\mathbf{k}, 0)$ is the Fourier-image of polarization distribution on the surface given by Eq. (8) or (9) at $z=0$. Eq. (14) is derived for rather prolate domain structures (e.g. domains with radius $r<<l$ ). In the adopted effective point charge model of the probe electric field, the OTF components $\widetilde{W}_{3 i j}(q)$ are [23]:

$\widetilde{W}_{333}(q)=-V \cdot d^{2} \int_{0}^{\infty} k d k \int_{0}^{2 \pi} d \psi \exp (-k d) \times$

$\times\left(\begin{array}{l}\frac{1}{\gamma \sqrt{k^{2}+q^{2}-2 k q \cos \psi}+k}+ \\ +\frac{\gamma \sqrt{k^{2}+q^{2}-2 k q \cos \psi}}{\left(\gamma \sqrt{k^{2}+q^{2}-2 k q \cos \psi}+k\right)^{2}}\end{array}\right) \approx$

$\approx-V\left(\frac{\gamma q d}{2}+\frac{(1+\gamma)^{2}}{1+2 \gamma}\right)^{-1}$,

$\widetilde{W}_{313}(q)=-V \cdot d^{2} \int_{0}^{\infty} k d k \int_{0}^{2 \pi} d \psi \exp (-k d) \times$

$\times\left(\begin{array}{l}\frac{1+2 v}{\gamma \sqrt{k^{2}+q^{2}-2 k q \cos \psi}+k}- \\ -\frac{\gamma \sqrt{k^{2}+q^{2}-2 k q \cos \psi}}{\left(\gamma \sqrt{k^{2}+q^{2}-2 k q \cos \psi}+k\right)^{2}}\end{array}\right) \approx$

$\approx-V\left(-\left(\gamma q d+\frac{(1+\gamma)^{2}}{\gamma}\right)^{-1}+\frac{(1+2 v)}{\gamma q d+1+\gamma}\right)$,

$$
\begin{aligned}
& \widetilde{W}_{351}(q)=-V \cdot d^{2} \int_{0}^{\infty} k d k \int_{0}^{2 \pi} d \psi \frac{\exp (-k d)}{k}+ \\
& +\frac{\gamma^{2}\left(k^{2}-k q \cos \psi\right)}{\left(\gamma \sqrt{k^{2}+q^{2}-2 k q \cos \psi}+k\right)^{2}} \approx \\
& \approx-V\left(\frac{\gamma(q d)^{3}}{6}+2(q d)^{2}+\frac{16-15 \gamma^{2}}{4 \gamma} q d+\frac{(1+\gamma)^{2}}{\gamma^{2}}\right)^{-1},
\end{aligned}
$$

where $d$ is effective charge-surface separation, $\sqrt{q_{1}^{2}+q_{2}^{2}}=q$. Here, approximation Eq. (15c) is valid for $\gamma<1$, whereas for $\gamma \geq 1$ the linear term $\sim q d$, which caused unphysical pole, should be omitted (as the result, the accuracy of Pade approximation decreases).

Using Eqs. (14), (8) and (2), effective piezoresponse $d_{33}^{\text {eff }}(x, y)=u_{3}(x, y) / V$ can be written as:

$d_{33}^{e f f}(x, y, V)=d_{0}^{e f f}(x)+2 \varepsilon_{0} \int_{0}^{\infty} k \mathrm{~J}_{0}\left(k \sqrt{x^{2}+y^{2}}\right) \times$

$\times\left(\widetilde{W}_{333}(k) \varepsilon_{33} Q_{11}+\widetilde{W}_{313}(k) \varepsilon_{33} Q_{12}+\right.$

$\left.+\widetilde{W}_{351}(k) \varepsilon_{11} Q_{44}\right) \frac{\widetilde{p}(\mathbf{k}, 0)}{V} d k$,

where $J_{0}$ is the Bessel function of zero order, $d_{0}^{\text {eff }}(x)$ is the bias-independent PFM profile of the flat $180^{\circ}$ domain wall calculated in details in Ref. [10]. The bias dependence of $\tilde{p}(\mathbf{k}, 0) \sim P_{V}$ is given by Eq. (10).

Below the probe apex, $x=y=0$, let us compare piezoresponse (17) calculated within LGD model with the expressions obtained within rigid approximation (RA) of the atomically-sharp domain walls, proposed in Refs. $[8,9,23]$. In the rigid approximation the domain shape was regarded as a half ellipsoid with the small and large axis equal to $r$ and $l$, respectively. The corresponding effective piezoresponse, measured in the vicinity of the spike-like or cylindrical domain can be approximated as:

$$
\begin{aligned}
& d_{33}^{\text {eff }}(0,0, V) \approx d_{0}^{\text {eff }}-\frac{3}{4}\left(d_{33}+\left(\frac{1}{3}+\frac{4}{3} v\right) d_{31}\right) \times \\
& \times \frac{\pi d-8 \sqrt{r^{2}-a^{2}}}{\pi d+8 \sqrt{r^{2}-a^{2}}}-\frac{d_{15}}{4} \frac{3 \pi d-8 \sqrt{r^{2}-a^{2}}}{3 \pi d+8 \sqrt{r^{2}-a^{2}}} .
\end{aligned}
$$

Here $r(V)$ is the domain radius and $a(V)$ is the shift of the probe axes with respect to the domain one, whose bias dependence is determined from the minimum of the corresponding free energy functional $[8,9] ; d$ is the effective charge - surface separation, dielectric anisotropy is small, $\varepsilon_{11} \approx \varepsilon_{33}$. Eq. (17) is valid for small shifts $2|a|<r$. The approximate analytical expression for the profile $d_{0}^{\text {eff }}(0)$ of an atomically-sharp $180^{\circ}$ domain wall was found in Ref. [23] as $d_{33}^{\text {eff }}(0) \approx$ 

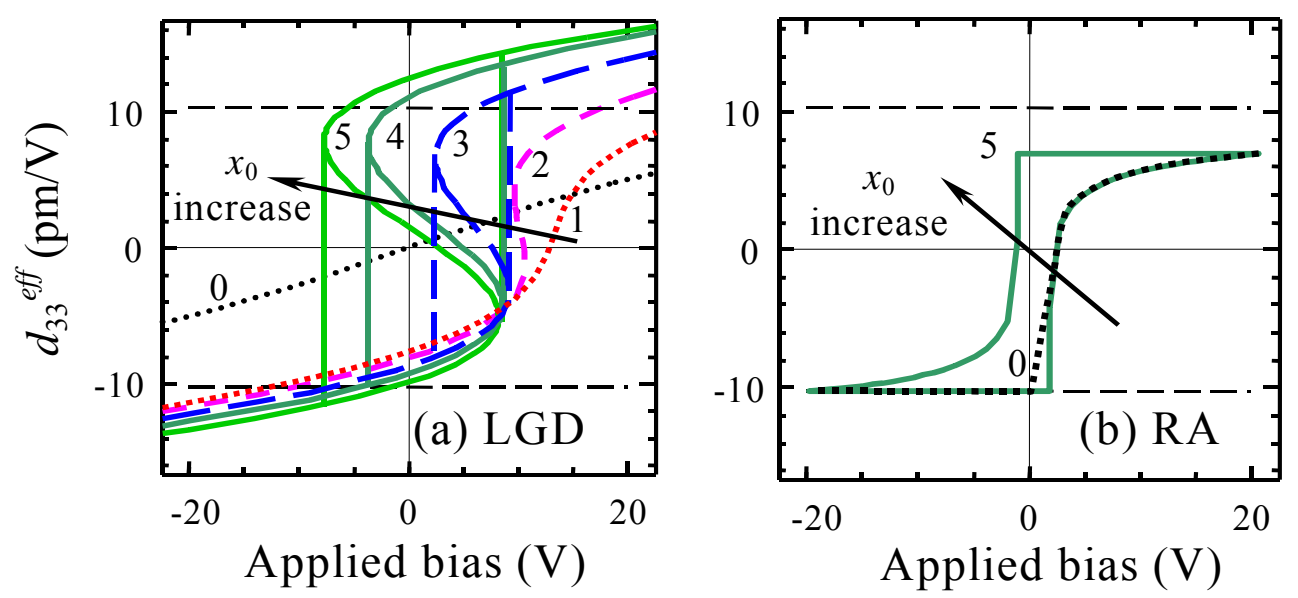

Fig. 5. Effective piezoelectric response vs. applied bias (PFM loops) for different distances $x_{0}$ from the domain wall for different $x_{0}=0,30,40,60,100,300 \mathrm{~nm}$ (curves 0, 1, 2, 3, 4, 5). Loops (a) are calculated from Eq. (16) within LGD approach, loops (b) are calculated from Eq. (17) within rigid approximation (RA). Effective distance $d=25 \mathrm{~nm}$. The material parameters for $\mathrm{LiNbO}_{3}$ are $\varepsilon_{11}=84, \alpha=-2 \cdot 10^{9}$ SI units, $P_{S}=0.75 \mathrm{C} / \mathrm{m}^{2}$. Poisson ratio is $v=0.3$; electrostriction coefficients are $Q_{44}=0.038, Q_{12}=-0.003, Q_{11}=0.016 \mathrm{~m}^{4} / \mathrm{C}^{2} ; L_{\perp}=1 \mathrm{~nm}$.

$\approx \frac{3}{4}\left(d_{33}+\left(\frac{1}{3}+\frac{4}{3} v\right) d_{31}\right) \frac{-x_{0}}{\left|x_{0}\right|+d / 4}+\frac{d_{15}}{4} \frac{-x_{0}}{\left|x_{0}\right|+3 d / 4}$,

where $x_{0}$ is the domain wall position with respect to the probe apex.

The effective piezoelectric response vs. applied bias calculated from Eq. (16) within LGD approach in comparison with rigid approximation (17) is shown in Fig. 5 for different distances from the domain wall.

It is clear from Fig. 5b that Eq. (16) reproduces the main features of the ferroelectric hysteresis (and so the PFM loops) far from the domain wall (i.e. bistability between the state with a single domain wall and the state with a nascent domain is possible at $\left|x_{0}\right| \widetilde{>} d$ ). This is a direct consequence of LGD model adopted here (as opposed to the rigid ferroelectric model with anomalous loop shape).

\section{Summary}

In the paper analytical Landau-Ginzburg-Devonshire theory was adopted for description of the domain structure formation by using the Scanning Probe Microscopy. We calculated effective piezoresponse and studied the depth profile of polarization distribution. It was shown that depending on the material parameters such as intrinsic domain wall width, the shape of equilibrium domains, induced by the probe, can be either oblate or prolate. The derived analytical expressions are valid for both the first and second order ferroelectrics.

\section{Acknowledgements}

Authors gratefully acknowledge financial support from National Academy of Science of Ukraine, joint RussianUkrainian grant NASU N 17-Ukr a (RFBR N 08-0290434).

\section{References}

1. G. Rosenman, P. Urenski, A. Agronin, Y. Rosenwaks, M. Molotski, Submicron ferroelectric domain structures tailored by highvoltage scanning probe microscopy // Appl. Phys. Lett. 82 (2), p. 103-105 (2003).

2. A.L. Kholkin, I.K. Bdikin, V.V. Shvartsman, A. Orlova, D.A. Kiselev, and A.A. Bogomolov, Local electromechanical properties of ferroelectric materials for piezoelectric applications, In: Scanning-Probe and Other Novel Microscopies of Local Phenomena in Nanostructured Materials, edited by S.V. Kalinin, B. Goldberg, L.M. Eng, and B.D. Huey // Mater. Res. Soc. Symp. Proc. 838E. Warrendale, PA, 2005, O7.6.

3. P. Paruch, T. Giamarchi, T. Tybell, and J.M. Triscone, Nanoscale studies of domain wall motion in epitaxial ferroelectric thin films. // $J$. Appl. Phys. 100 (5), 051608 (2006).

4. T.J. Yang, V. Gopalan, P.J. Swart, and U. Mohideen, Direct observation of pinning and bowing of a single ferroelectric domain wall // Phys. Rev. Lett. 82 (20), p. $4106-4109$ (1999).

5. M. Molotskii, A. Agronin, P. Urenski, M. Shvebelman, G. Rosenman, Y. Rosenwaks, Ferroelectric domain breakdown // Phys. Rev. Lett. 90 (10), 107601 (2003).

6. S.V. Kalinin, A. Gruverman, B.J. Rodriguez, J. Shin, A.P. Baddorf, E. Karapetian, M. Kachanov, Nanoelectromechanics of polarization switching in piezoresponse force microscopy // J. Appl. Phys. 97 (7), 074305 (2005).

7. M. Molotskii, M. Shvebelman, Dynamics of ferroelectric domain formation in an atomic force microscope // Phyl. Mag. 85, p. 1637-1655 (2005). 
8. A.N. Morozovska, S.V. Svechnikov, E.A. Eliseev, S. Jesse, B.J. Rodriguez, S.V. Kalinin, Piezoresponse Force Spectroscopy of ferroelectric-semiconductor materials // J. Appl. Phys. 102 (11), 114108 (2007).

9. A.N. Morozovska, S.V. Svechnikov, E.A. Eliseev, B.J. Rodriguez, S. Jesse, S.V. Kalinin, Local polarization switching in the presence of surface charged defects: microscopic mechanisms and Piezoresponse Force Spectroscopy observations // Phys. Rev. B 78 (5), 054101 (2008).

10. A.N. Morozovska, E.A. Eliseev, G.S. Svechnikov, V. Gopalan, and S.V. Kalinin, Effect of the intrinsic width on the Piezoelectric Force Microscopy of a single ferroelectric domain wall // J. Appl. Phys. 103 (12), 124110 (2008).

11. A.N. Morozovska, S.V. Kalinin, E.A. Eliseev, V. Gopalan, and S.V. Svechnikov, The interaction of an 180-degree ferroelectric domain wall with a biased Scanning Probe Microscopy tip: effective wall geometry and thermodynamics in GinzburgLandau-Devonshire theory // Phys. Rev. B 78 (12), 125407 (2008).

12. F. Felten, G.A. Schneider, J.M. Saldaña, and S.V. Kalinin, Modeling and measurement of surface displacements in $\mathrm{BaTiO}_{3}$ bulk material in piezoresponse force microscopy // J. Appl. Phys. 96 (1), p. 563-568 (2004).

13. D.A. Scrymgeour and V. Gopalan, Nanoscale piezoelectric response across a single antiparallel ferroelectric domain wall // Phys. Rev. B 72 (2), 024103 (2005).

14. A.I. Lur'e, Three-dimensional Problems of the Theory of Elasticity. Interscience Publishers, 1964.

15. L.D. Landau and E.M. Lifshitz, Theory of Elasticity, Theoretical Physics, Vol. 7. Butterworth-Heinemann, Oxford, U.K., 1998.
16. A.K. Tagantsev, and G. Gerra, Interface-induced phenomena in polarization response of ferroelectric thin films // J. Appl. Phys. 100 (5), 051607 (2006).

17. C.H. Woo and Yue Zheng, Depolarization in modeling nano-scale ferroelectrics using the Landau free energy functional // Appl. Phys. A 91, p. 59-63 (2007).

18. V.A. Zhirnov, A contribution to the theory of domain walls in ferroelectrics // Zh. Eksp. Theor. Fiz. 35 (5), p. 1175-1180 (1959) (in Russian) [Sov. Phys. JETP 8, p. 822 (1959)].

19. W. Cao, and L.E. Cross, Theory of tetragonal twin structures in ferroelectric perovskites with a firstorder phase transition // Phys. Rev. B 44 (1), p. 5-12 (1991).

20. R. Kretschmer, and K. Binder, Surface effects on phase transition in ferroelectrics and dipolar magnets // Phys. Rev. B 20 (3), p. 1065-1076 (1979).

21. M.E. Lines and A.M. Glass, Principles and Application of Ferroelectrics and Related Materials. Clarendon Press, Oxford, 1977.

22. S. Choudhury, Y. Li, N. Odagawa, Aravind Vasudevarao, L. Tian, P. Capek, V. Dierolf, A.N. Morozovska, E.A. Eliseev, S.V. Kalinin, Y. Cho, L-Q. Chen, V. Gopalan, The influence of $180^{\circ}$ ferroelectric domain wall width on the threshold field for wall motion // J. Appl. Phys. 104 (8), 084107 (2008).

23. A.N. Morozovska, E.A. Eliseev, S.L. Bravina and S.V. Kalinin, Resolution function theory in Piezoresponse Force Microscopy: domain wall profile, spatial resolution, and tip calibration // Phys. Rev. B 75 (17), 174109-1-18 (2007). 